\title{
«L'usurpateur boiteux » se met en marche : évolutions de la tussentaal flamande
}

The 'usurpateur boiteux' is on the move: evolutions in Flemish Tussentaal

\section{Gudrun Ledegen}

\section{OpenEdition}

1 Journals

Édition électronique

URL : https://journals.openedition.org/lbl/559

DOI : $10.4000 / \mathrm{lbl} .559$

ISSN : 2727-9383

Éditeur

Université de Bretagne Occidentale - UBO

\section{Édition imprimée}

Date de publication : 30 décembre 2019

Pagination : 101-118

ISBN : 979-10-92331-49-3

ISSN : $1270-2412$

Référence électronique

Gudrun Ledegen, « "L'usurpateur boiteux » se met en marche : évolutions de la tussentaal flamande », La Bretagne Linguistique [En ligne], 23 | 2019, mis en ligne le 01 mai 2020, consulté le 03 mai 2021. URL : http://journals.openedition.org/lbl/559; DOI : https://doi.org/10.4000//bl.559

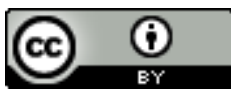

La Bretagne Linguistique est mise à disposition selon les termes de la Licence Creative Commons Attribution 4.0 International. 


\title{
«L'usurpateur boiteux » se met en marche : évolutions de la tussentaal flamande
}

\author{
GUDRUN LEDEGEN*
}

\section{Introduction}

Pour les Hollandais-en-Flandres, ce que tous les Flamands parlent [comme langue] est un problème. Je penserais que ce n'est pas ce que tout le monde mais ce que personne ne parle qui serait un problème. Que tant de Flamands parlent et écrivent une langue que personne d'autre n'utilise, même pas en Flandres, c'est de ça qu'il nous faut nous occuper ${ }^{1}$.

\begin{abstract}
Cet article traite de la variété substandard flamande, nommée tussentaal ('langue intermédiaire'), son évolution depuis la deuxième guerre mondiale et sa récente affirmation, s'inscrivant dans les analyses sur la substandardisation et la standardisation. Après une présentation des terminologies et concepts nécessaires pour traiter de la problématique de la tussentaal en Flandres, je présenterai des éléments portant sur l'histoire de la standardisation du néerlandais et exemplifierai des traits linguistiques particuliers à cette région. Cet écrit est aussi un hommage à Kas Deprez, un des seuls sociolinguistes belges
\end{abstract}

\footnotetext{
* Professeure des Universités en Sciences du Langage / Sociolinguistique.

Co-Directrice du Laboratoire PREFICS - EA 7469. Université Rennes 2.
}

Je tiens à remercier les deux relecteurs anonymes du comité scientifique de La Bretagne Linguistique qui m'ont permis d'améliorer mon écrit et d'ouvrir de nombreuses nouvelles pistes à venir.

1. Kas DEPREZ, "Referentiekader voor het Vlaams Nederlands», De Standaard 9/10/1991, p. 9 : Voor de Nederlanders-in-Vlaanderen is wat alle Vlamingen zeggen een probleem. Ik zou denken dat niet wat iedereen maar wat niemand zegt een probleem is. Dat zoveel Vlamingen een taal spreken en schrijven die niemand anders gebruikt, ook in Vlaanderen niet, daar moeten we iets aan doen. [les traductions sont de ma main]. 
à poser l'existence d'un néerlandais belge standard dès les années 80 ; ses écrits et discours dans la société ont ouvert la voie aux changements de paradigme qui permettent non seulement aujourd'hui de poser le pluricentrisme du néerlandais, et l'existence d'un néerlandais belge standard, mais aussi l'émergence d'une pratique colloquiale qui pourrait à terme faire évoluer ce standard.

\section{Flamand de lotissement, tussentaal, ...}

Démarrons par une explication des termes du titre choisi pour cet article : il s'agit d'une référence double, d'une part à l'excellent ouvrage De manke usurpator. Over Verkavelingsvlaams ('L'usurpateur boîteux. Du flamand de lotissement') édité par Absillis, Jaspers \& Van Hoof (2012), qui constitue une référence sur les études récentes sur la tussentaal ('langue intermédiaire') et le standard en Flandres. D'autre part, à l'auteur de cette expression dénigrante qui désigne la langue informelle flamande, située entre les dialectes et le standard, Geert van Istendael. Cet auteur de Het Belgisch labyrint of De schoonheid van wanstaltigheid. ('Le labyrinthe belge ou La beauté de la difformité') (1989) décrit comme suit la nouvelle pratique flamande, qu'il abhorre :

Il y a quelque chose de nouveau, quelque chose de sale en train d'attaquer la langue dans les Pays-Bas du sud [i.e. la Belgique]. Un usurpateur boiteux sans vêtements, mais il a le caractère vaniteux et la lourdeur d'un parvenu. Il s'appelle flamand de lotissement. C'est la langue qui est parlée dans les villas huppées sur les terres remembrées de nos villages mutilés. C'est la langue de garçons et de filles qui vont dans une école respectable et se moquent des autres enfants parce qu'ils parlent si grossièrement ${ }^{2}$.

Ce terme «flamand de lotissement», proposé par van Istendael, a connu un grand succès ; il est d'ailleurs entré en 1999 dans le Van Dale, Groot woordenboek van de Nederlandse taal ('Van Dale, Grand dictionnaire de la langue néerlandaise'), avec comme définition : "la

2. Geert VAN ISTENDAEL, Het Belgisch labyrint of De schoonheid van wanstaltigheid, Amsterdam, Arbeiderspers, 1989, p. 15 : Er is iets nieuws, iets vuils de taal in de zuidelijke Nederlanden aan het aantasten. Een manke usurpator in kale kleren, maar hij heeft de verwaandheid en de lompheid van een parvenu. Hij heet Verkavelingsvlaams. Het is de taal die gesproken wordt in de betere villa's op de verkavelde grond van onze verminkte dorpen. Het is de taal van de jongens en de meisjes die naar een deftige school gaan en andere kinderen uitlachen omdat die zo onbeschaafd praten. 
langue orale informelle qui en Belgique néerlandophone n'est pas le dialecte mais pas non plus le néerlandais général ${ }^{3}$ ».

Les linguistes professionnels aussi ont adopté ce terme très facilement ${ }^{4}$, le signe d'une normativité à l'égard de cette pratique qui était et reste encore souvent virulente. Mais aussi le signe d'une difficulté à nommer le phénomène et à le cerner : le tableau ci-dessous montre quelques termes scientifiques versus des discours portant des jugements normatifs qui circulent dans la société :

\begin{tabular}{|l|l|}
\hline Concepts scientifiques & Discours épilinguistiques négatifs \\
\hline tussentaal ('langue intermédiaire, interlangue') & $\begin{array}{l}\text { Verkavelingsvlaams ('flamand de lotissement') } \\
\text { soapvlaams ('flamand de soap') } \\
\text { hamburgertaal ('langue de hamburger') } \\
\text { sloddertaal ('langue malpropre') } \\
\text { koetervlaams ('flamand d'idiot') } \\
\text { nepdialect ('faux dialecte') } \\
\text { ontaal ('non-langue') }\end{array}$ \\
\hline
\end{tabular}

Tableau 1 - Termes désignant la langue néerlandaise orale informelle en Flandres ${ }^{6}$

Absillis, Jaspers \& Van Hoof signalent toutefois que certains linguistes décrivent et étudient ce que d'autres appellent cette « répugnante mi-langue ${ }^{7}$ ». Ils soulignent avec ironie que certains d'entre eux «ont même osé se demander si la haine pour [la tussentaal] n'était pas plus marquante comme phénomène que la tussentaal même ${ }^{8} \|^{9}$.

3. "informele spreektaal in Nederlandstalig België die geen dialect is maar ook geen Algemeen Nederlands". Le néerlandais standard était auparavant désigné par le terme ABN (Algemeen Beschaafd Nederlands, 'néerlandais poli/civilisé généralisé).

4. Tout aussi remarquable est que G. van Istendael ait reçu un doctorat honoris causa pour sa contribution d'avoir baptisé ainsi le substandard flamand.

5. Le terme schoon étant flamand, son équivalant hollandais est mooi.

6. Kevin Absillis, Jürgen Jaspers, \& Sarah Van Hoof (dir.), De manke usurpator. Over Verkavelingsvlaams, Gent, Academia Press, 2012, p. 4.

7. Id, p. 8.

8. Jeroen JASPERS, «Het Vlaamse stigma. Over tussentaal en normativiteit», Taal en Tongval, 53, 2001, p. 129-153; Johan DE CALUWE, "Tien stellingen over tussentaal in Vlaanderen", in Johan De Caluwe et al. (dir.), Taalvariatie en taalbeleid, AntwerpenApeldoorn, Garant, 2002, 57-67.

9. Id., p. 8. 
Le terme tussentaal est utilisé dans les études sociolinguistiques sur la substandardisation ${ }^{10}$ pour désigner cette pratique orale informelle qui n'a pas le statut d'un standard mais dont il s'avère que les fonctionnalités sont bien plus larges que celles des dialectes. Toujours est-il que le terme tussentaal (i.e. interlangue) provient initialement des recherches en didactique, désignant une norme visée vers laquelle tendent les productions d'apprenants de façon dynamique (Selinker, 1972); il s'avère ainsi impropre parce qu'il n'y a pas une langue à cibler, à atteindre, la tussentaal constituant la langue cible même ${ }^{11}$. En effet, le néerlandais standard est de plus en plus ressenti comme une variété artificielle imposée par en haut ${ }^{12}$.

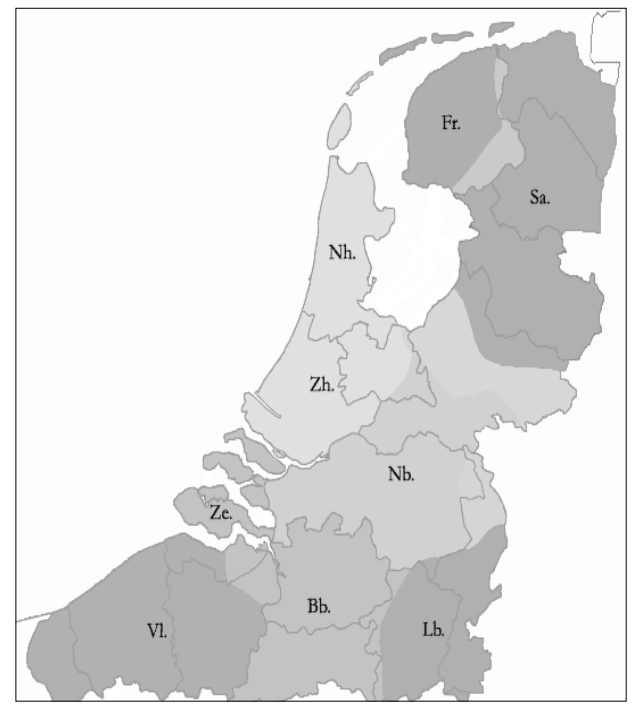

\begin{tabular}{|l|l|l|}
\hline Fr. & frison & \\
\hline Sa. & saxe & \\
\hline Nh. \& Zh. & hollandais (nord et sud) & Pays-Bas \\
\hline Nb. & brabant du nord & \\
\hline Ze. & zélandais & \\
\hline Bb. & brabant belge & \\
\hline Vl. & flamand (ouest \& est) & Belgique \\
\hline Lb. & limbourgeois & \\
\hline
\end{tabular}

Figure 1 - Répartition globale des dialectes et

régiolectes du néerlandais [d'après Hoppenbrouwers, 2001]

10. Jeroen JASPERS, Frank BRISARD, "Verklaringen van substandaardisering : tussentaal als gesitueerd taalgebruik", Leuvense Bijdrage, 2006, p. 35-70.

11. José CAJOT, "Waarom het Verkavelingsvlaams onvermijdelijk was. De ontwikkeling van een informele omgangstaal in Vlaanderen", in Kevin Absillis, Jürgen Jaspers \& Sarah Van Hoof (dir.), De manke usurpator. Over Verkavelingsvlaams, Gent, Academia Press, 2012, p. 49.

12. Jeroen JASPERS, Sarah VAN HOOF, "Ceci n'est pas une tussentaal. Evoking standard and vernacular language through mixed Dutch in Flemish telecinematic discourse», Journal of Germanic Linguistics, 27.1, 2015, p. 4. 
Il s'avère que, structurellement parlant, on puisse parler d'une forme soignée de régiolecte, encore variable par régions mais révélant des formes flamandes généralement attestées, avec les dialectes brabançons exerçant l'influence la plus grande. Ces derniers dialectes (indiqués en gras dans la légende ci-dessous) forment de fait la plus grande zone en Flandres :

Jusque dans les années 2000, peu de recherches portaient sur la description et l'explication de l'usage de la tussentaal flamande ; depuis, celles-ci ont enfin pu se développer scientifiquement, quittant les discussions polémiques, d'opinion et d'émotion. Absillis, Jaspers \& Van Hoof ${ }^{13}$ listent ainsi 15 références essentielles dont les années s'étalent ainsi :

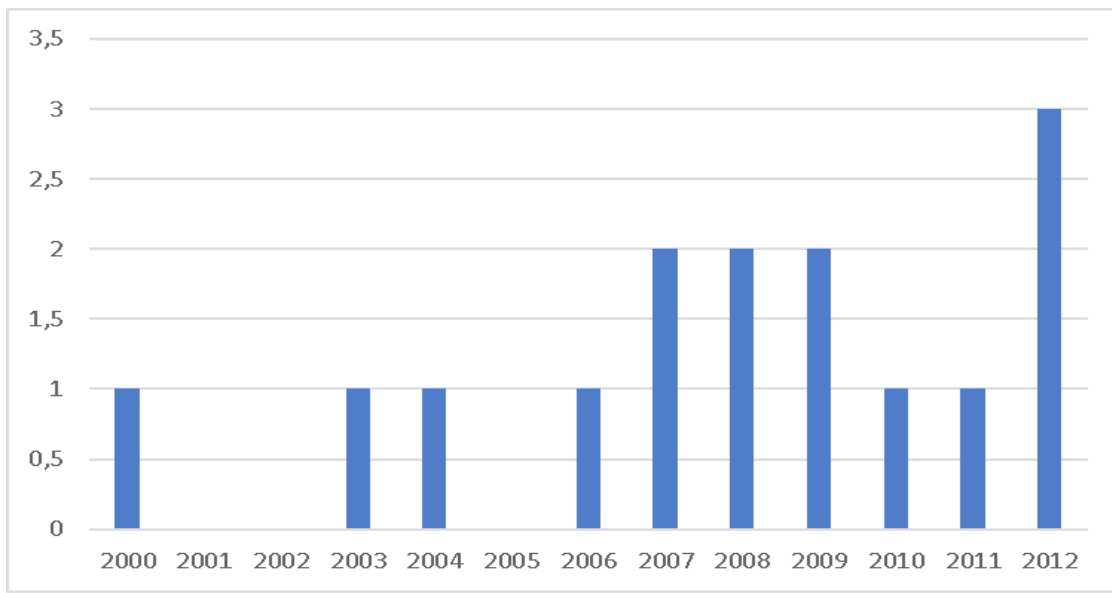

Figure 2 - Publications essentielles consacrées à la tussentaal par année

Pourtant, dès les années 80, Kas Deprez, professeur de sociolinguistique à l'Université d'Anvers et de Louvain, a mené des recherches sociolinguistiques qui montraient que les Flamands ne voulaient pas parler comme les Néerlandais ${ }^{14}$. En effet, les différences

13. Kevin ABSILlis, Jürgen JASPERS, Sarah VAN HOOF (dir.), De manke usurpator. Op. cit.

14. E.A. Kas DepreZ, Naar Een Eigen Identiteit. Resultaten En Evaluatie Van Tien Jaar Taalsociologisch En Sociolinguistisch Onderzoek Betreffende De Standaardtaal In 
culturelles, politiques et linguistiques étant clairement attestées et ressenties, Deprez appelait à un néerlandais plus belge ou flamand, incluant de la tolérance pour les gallicismes et purismes. Mais le monde universitaire ne voulait pas d'une modification du jeu normatif, où ses propres membres constituaient de fait l'élite linguistique : un collègue de sa propre université considérait ainsi Kas Deprez comme "particulièrement controversé, attaquable et dangereux ${ }^{15}$ ".

A contrario, Koen Jaspaert appuie les recherches de Kas Deprez en suggérant que les Flamands feraient mieux d'arrêter de conformer leur usage linguistique aux normes néerlandaises du nord : qui ne veut être damné à toujours courir après le standard, ferait mieux de faire l'inverse, c'est-à-dire "adapter ses propres normes à son propre usage linguistique ${ }^{16}$ », mais il est un des rares dans ces années-là à soutenir une telle proposition.

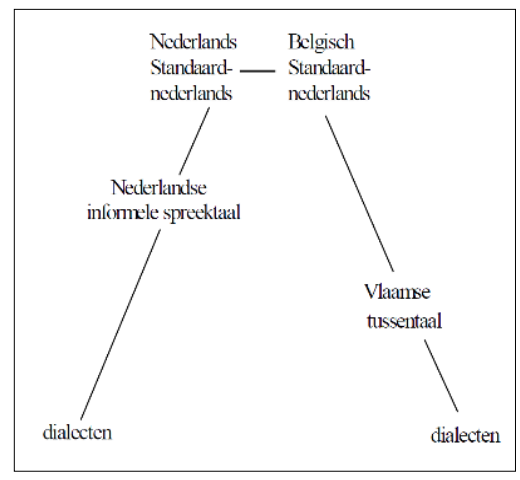

Figure 3 - Différences entre le néerlandais aux Pays-Bas et en Belgique $^{17}$

Vlaanderen. Onuitgegeven Proefschrift, Katholieke Universiteit Leuven, 1981 ; Kas Deprez, Geerts GuIDO, "Lexical And Pronominal Standardization. The Evolution Of Standard Netherlandic In West-Flanders ", in Zeitschrift Für Dialektologie Und Linguistik, Beihefte Neue Folge, 22, 1977 ; Voir Aussi Jean LE DÛ, Gudrun Ledegen, Pour Un Néerlandais De Belgique. Écrits D’un Précurseur. Hommage À Kas Deprez, Paris, L'Harmattan, Coll. «Espaces Discursifs», 2019.

15. "Bijzonder controversieel [...] aanvechtbaar en riskant», (R. GEERAERTS, «Deprez-historie van het Vlaams», Yang, 37.4, 2001, p. 656).

16. "[zijn] eigen normen aan [zijn] taalgebruik aanpassen", (K. JASPAERT, 1989. "Van oude normen, de dingen die voorbij gaan. Over de Nederlands-Vlaamse taalverhouding", Kultuurleven, 56.8, p. 16-23).

17. D. GEERAERTS, «Een zondagspak», Ons Erfdeel, 3 (44), 2001, p. 339. Légende : 1. néerlandais standard néerlandais ; 2 . langue parlée informelle néerlandaise ; 3 . dialectes ; 4. néerlandais standard belge ; 5 . langue intermédiaire belge ; 6. dialectes, p. 339. 
La figure 3 résume bien la situation pluricentrique ${ }^{18}$ du néerlandais avec deux centres en interaction:

Jusqu'à récemment, cette présentation était encore considérée comme problématique, parce que la norme linguistique était située aux Pays-Bas : sa variété nationale fonctionnait comme le modèle officiel du néerlandais standard en Belgique. En effet, jusqu'en 1932 le néerlandais ne fonctionnait guère comme langue nationale et cultivée en Flandres : le français était utilisé par les classes supérieures, dominant l'éducation, l'administration, la politique et la vie publique en général. Et la population parlait les variétés régionales de néerlandais : aucune variété suprarégionale de néerlandais n'émergeait ainsi en Flandres, parce que le français y occupait cette place. Ainsi, la diglossie classique, avec le français comme variété haute et le flamand comme variété basse, était en place. Et quand il y a eu besoin d'un standard pour le néerlandais, quand celui-ci devient langue officielle en Flandres, la question s'est posée d'adopter un mouvement 'particularisant' vs mouvement 'intégrationniste' ; elle s'est soldée par l'adoption du néerlandais standard des Pays-Bas ${ }^{19}$.

Toutefois, en dépit de plus d'un demi-siècle de politique linguistique promouvant la convergence avec le néerlandais du Nord, une divergence de plus en plus grande est attestée : ainsi, les jeunes générations ont une préférence plus grande pour les variantes (syntaxiques) endogènes, brabançonnes, que les anciennes génération ${ }^{20}$ (cf. ci-dessous).

18. ClYne M., "Pluricentricity: National Variety ", in U. Ammon (dir.), Status and Function of Languages and Language Varieties, Berlin-New York, Mouton de Gruyter, 1989, p. 357-371.

19. Pour une présentation de l'histoire sociolinguistique de la Flandre, Gudrun LEDEGEN, "Pour un néerlandais belge. Ecrits d'un précurseur. Introduction", in Jean Le Dû, Gudrun Ledegen, Pour un néerlandais de Belgique. Écrits d'un précurseur Hommage À Kas Deprez, Paris, L'Harmattan, Coll. «Espaces discursifs», 2019, p. 13-23.

20. Les informateurs jeunes sont nés entre 1967 et 1982 ; les informateurs anciens entre 1938 et 1956. Reinhild VANDEKERCKHOVE, "Belgian Dutch versus Netherlandic Dutch : New patterns of divergence? On pronouns of address and diminutives", Multilingua, 24, 2005, p. 379-397. 


\section{Descriptions fonctionnelles et linguistiques de la tussentaal}

Les descriptions fonctionnelles montrent que la tussentaal remplace le néerlandais standard dans des domaines de plus en plus larges, et qu'elle se développe comme le standard flamand informel ${ }^{21}$, utilisé autant voire davantage par les jeunes que par les plus âgés, autant par les peu scolarisés que par les personnes hautement instruites. Les analyses de Koen Plevoets datent ainsi l'orientation vers davantage de tussentaal à partir de la génération née dans les années $1970^{22}$.

Certes, l'usage reste une variété avant tout parlée, et à l'écrit il se limite avant tout aux nouveaux médias mais la publicité y puise de plus en plus (cf. plus bas); la tussentaal semble en marche vers une standardisation, de facto. Ainsi, la tussentaal ne doit pas être considérée comme un phénomène de transition ou une phase vers un «meilleur» néerlandais. Comme le dit J. Cajot, elle n'est pas «un sous-produit non voulu, le résultat raté d'un processus de standardisation non abouti ou un déraillement accidentel» : «Geen accident de parcours, maar onvermijdelijk ${ }^{23}$ " ('pas un accident de parcours mais (un fait) inévitable').

Pour ce qui est des descriptions linguistiques, elles révèlent ${ }^{24}$ :

- une divergence sur le plan phonologique

- une convergence sur le plan lexical

- une divergence sur le plan syntaxique

Pour le plan phonologique, la carte ci-dessous illustre clairement que les zones périphériques présentent les distances ${ }^{25}$ les plus grandes du néerlandais standard, et tout particulièrement toute la Flandre :

21. Kevin AbSILlis, Jürgen JASPERS, Sarah VAN HOOF, op. cit., 2012, p. 26.

22. Koen Plevoets, Tussen spreek-en standaardtaal. Een corpusgebaseerd onderzoek naar de situationele, regionale en sociale verspreiding van enkele morfosyntactische verschijnselen uit het gesproken Belgisch-Nederlands, Leuven, Dissertation Doctorale, 2008, p. 177-179.

23. José CAJOT, op. cit., p. 52.

24. Id., p. $40 \mathrm{sv}$.

25. Hoppenbrouwers (1988) a calculé les distances de 156 dialectes distincts par rapport au néerlandais standard, à partir de caractéristiques phonologiques. Son étude est basée sur la fréquence avec laquelle des items phonologiques sont utilisés dans différents dialectes dans un discours libre transcrit en API (feature frequency method, FFM). 


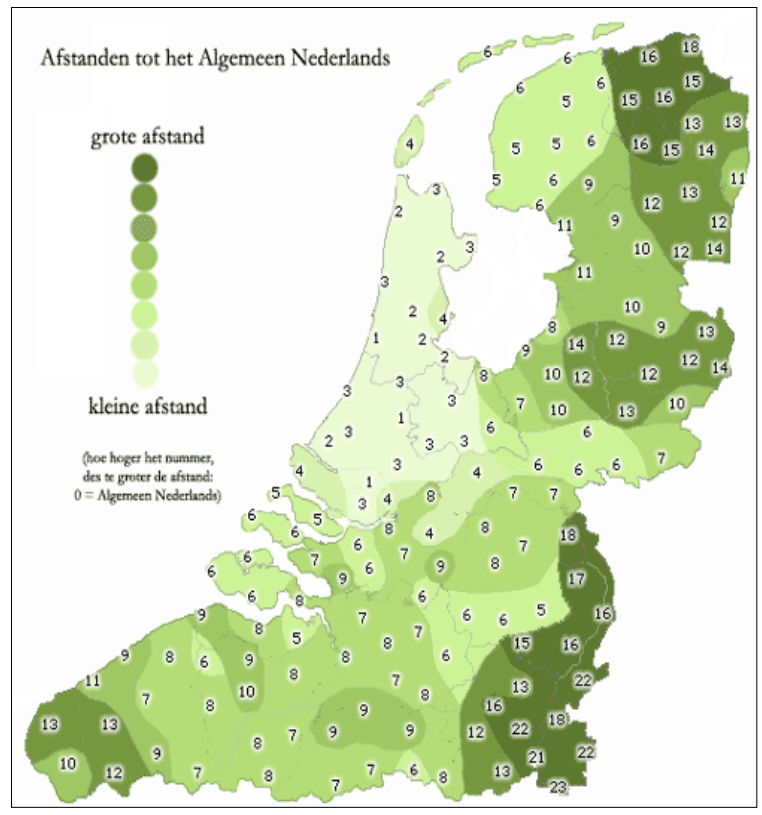

Figure 4 - Distances chiffrées du néerlandais standard ( 0 = néerlandais standard). [Hoppenbrouwers, 1988]

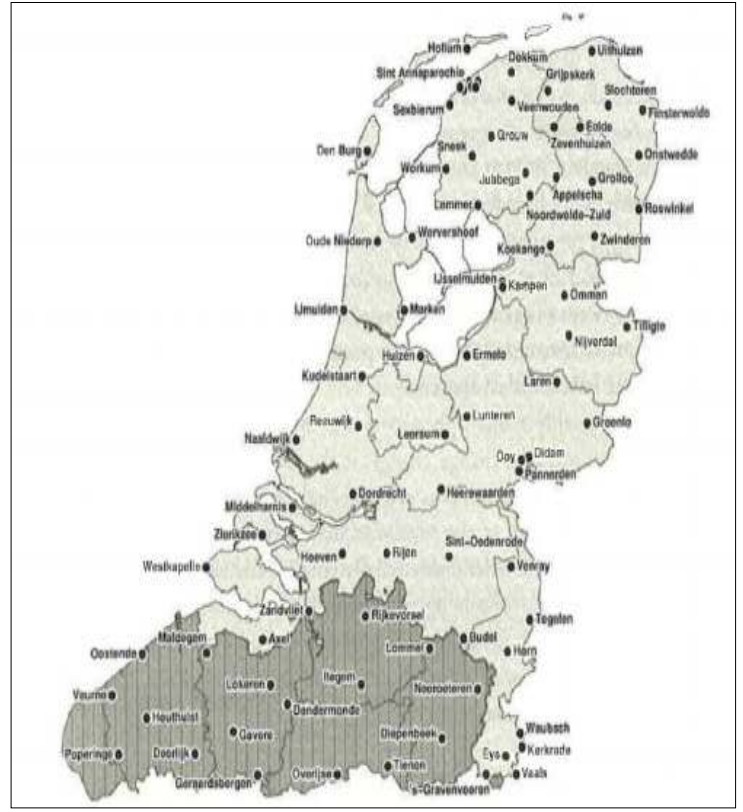

Figure 5 - Carte de variétés dialectales du néerlandais (les provinces des Pays-Bas en gris et de la Belgique en gris foncé rayé). [Heeringa \& Hinskens, 2014] 
Sur le plan syntaxique, différents traits contrastent le néerlandais des Pays-Bas et celui de la Belgique ${ }^{26}$; nous mentionnons ici le plus représentatif, l'usage aux Pays-Bas (P-B) et en Belgique (B) pour ce qui est des pronoms sujet et objet de $2^{\mathrm{e}}$ personne ${ }^{27}$ :

\begin{tabular}{|l|c|c|c|}
\hline & sujet & objet & possessif \\
\hline $\begin{array}{l}\text { néerlandais P-B } \\
\text { INFORMEL }\end{array}$ & $j e / j i j$ & $j e / j o u$ & $j e / j o u w$ \\
\hline $\begin{array}{l}\text { néerlandais P-B } \\
\text { FORMEL }\end{array}$ & $U$ & $u$ & $u w$ \\
\hline $\begin{array}{l}\text { néerlandais (colloquial) B } \\
\text { INFORMEL + FORMEL }\end{array}$ & $g e / g i j$ & $U$ & $u w$ \\
\hline
\end{tabular}

Tableau 2 - Pronoms sujet et objet $2^{\mathrm{e}}$ personne Pays-Bas/Belgique ${ }^{28}$

Les exemples suivants éclairent cette répartition :

(1) 'Tu dors beaucoup' SUJET
(a) néerlandais standard informel (P-B) JE slaapt veel
(b) néerlandais standard formel (P-B) U slaapt veel
(c) néerlandais informel \& formel (B) GE slaapt veel

(2) 'Je t'ai vu hier' OBJET
(a) néerlandais standard informel (P-B) ik zag JE gisteren
(b) néerlandais standard formel (P-B) ik zag U gisteren
(c) néerlandais informel \& formel (B) ik zag U gisteren

Il est à noter que selon les régions en Flandre, cet usage de ge/gij en tant que pronom sujet présente des variations :

26. W. HAESERYN, "Grammaticale verschillen tussen het Nederlands in België en het Nederlands in Nederland: een poging tot inventarisatie", in Van Hout, R. \& J. Kruijsen (dir.), Taalvariaties. Toonzettingen en modulaties op een thema, Dordrecht, Foris, 1996, p. 109-126.

27. Reinhild VANDEKERCKHOVE, art. cit.

28. Idem. 


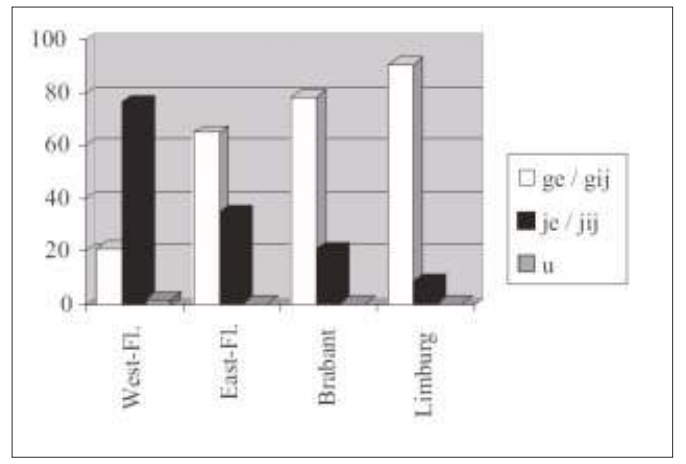

Figure 6 - Pronoms sujet selon les régions en Flandre ${ }^{29}$

Ainsi, la majorité des régions attestent ge/gij; seule la région des Flandres de l'Ouest emploie je/jij, parce que ce trait syntaxique figure dans son propre dialecte, comme on peut le voir dans les vœux ouestflamands de l'année 2019 ci-dessous :

\begin{tabular}{|l|l|l|}
\hline Flandres de l'Ouest & néerlandais standard & Traduction \\
\hline ojmoleutet & Als je maar plezier hebt & Que tu aies du plaisir \\
\hline ojmotietet & Als je maar tijd hebt & Que tu aies du temps \\
\hline ojmogoestet & $\begin{array}{l}\text { Als je maar smaak hebt } \\
\text { (goest ou goesting }<\text { goût })\end{array}$ & Que tu aies du goût \\
\hline ojmosjanset & $\begin{array}{l}\text { Als je maar geluk hebt } \\
\text { (sjans }<\text { chance })\end{array}$ & Que tu aies de la chance \\
\hline ojmoginambraset & $\begin{array}{l}\text { Als je maar geen ruzie hebt } \\
\text { (ambras }<\text { embrasser })\end{array}$ & Que tu n'aies pas de querelles \\
\hline ojmoruptitrustet & Als je maar op tijd rust hebt & Que tu aies du repos à temps \\
\hline ojmogindustet & Als je maar geen dorst hebt & Que tu n'aies pas soif \\
\hline ojmoginmoeitet & Als je maar geen moeite hebt & Que tu ne sois pas fatigué \\
\hline ojmogezoendziet & Als je maar gezond zijt & Que tu sois en bonne santé \\
\hline otmogoedis in 2019... & Als het maar goed is in 2019... & Que ce soit bien en 2019... \\
\hline
\end{tabular}

Tableau 3 - Réalisation du pronom sujet tu en ouest-flamand

29. Id. 
On constate une même différence pour les pronoms objet, où la majorité des régions atteste $u$ et les Flandres de l'Ouest lui préfèrent jel jou :

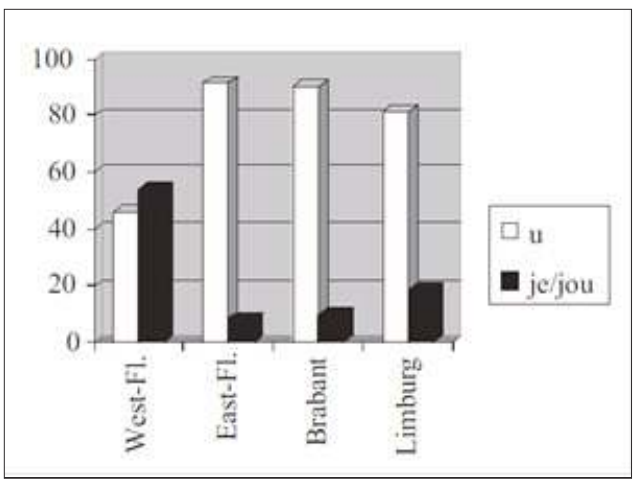

Figure 7 - Pronoms objet selon les régions en Flandre ${ }^{30}$

\section{Attestations dans la communication publique}

Le domaine de la communication publique fait volontiers appel à la tussentaal ${ }^{31}$ pour marquer un style naturel et informel ${ }^{32}$ : il en est ainsi pour la prévention routière :

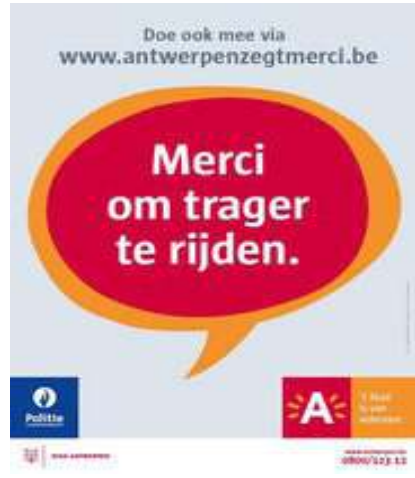

"Merci om trager te rijden" :

'Merci de rouler plus lentement'

30. Id.

31. Signalée en gras dans les exemples.

32. Kevin Absillis, Jürgen JaSPeRS \& Sarah VAN HoOf, op. cit., 2012, p. 5. 


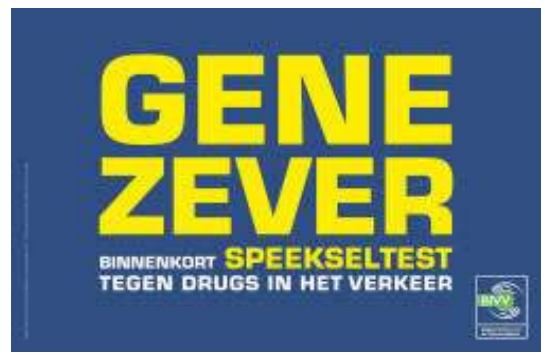

"Gene zever : binnenkort speekseltest tegen drugs in het verkeer" :

'Pas d'histoires/blabla (expression figée,

littéralement 'pas de salive') : bientôt test de salive contre les drogues dans la circulation routière'

$\mathrm{Ou}$ encore des affiches pour signaler l'emplacement des vélos libres (blue bikes) imitant des arrêts de train chez :

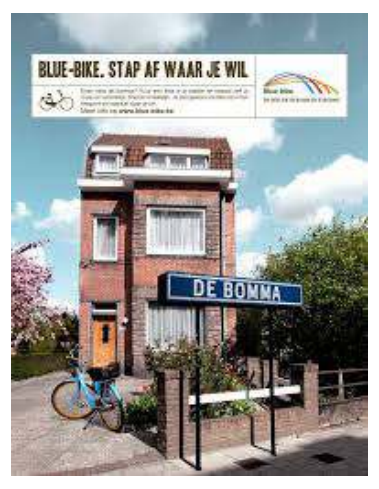

"De bomma" :

'La grand-mère' (< bonne-maman)

Et les informations estudiantines :

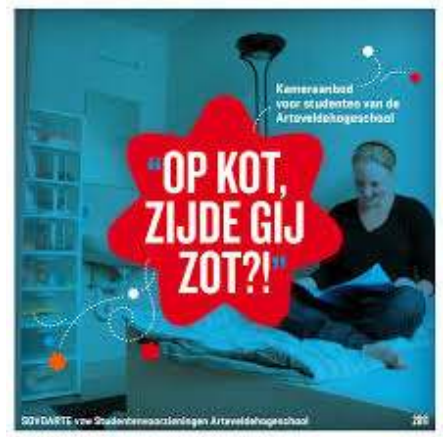

"Op kot, zijde gij zot ?!" :

'En chambre d'étudiant, tu es fou ?!' 
À noter que l'expression étudiante $k o t^{33}$ se combine ici avec la forme syntaxique ge qui relève de la tussentaal (à la différence de l'exemple précédent qui emploie je: "Stap af waar je will' : 'Descends où tu veux').

Ou encore, la publicité tout court :

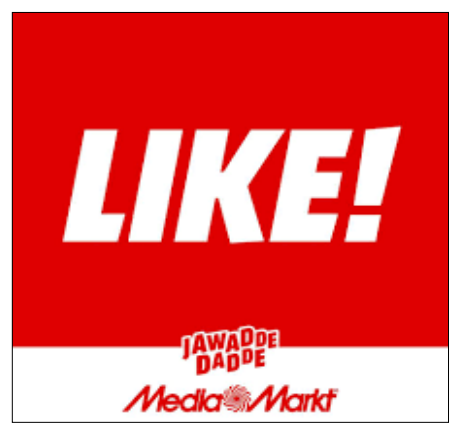

"Jawadde, dadde" :

'ça alors' (littéralement : oui quoi ça)

(publicité pour Mediamarkt, un hypermarché)

Pour finir cette présentation, notons la grande variabilité des pratiques qui peuvent glisser sur le continuum entre standard et dialecte : la tussentaal peut prendre appui sur le standard, quand un locuteur opte pour un vocabulaire et une structure phrastique standard, et troque son accent régional pour un accent de néerlandais général, mais qui laisse tout de même tomber le $-t$ final dans des mots comme dat ('ce'), wat ('que'), niet ('pas') et met ('avec'). Mais elle peut tout aussi bien être proche des dialectes, quand les locuteurs utilisent des mots non-standard comme tas ('tasse') au lieu de kopje, s'adressent à leur interlocuteur avec ge au lieu de je, accordent les adjectifs (accusatif) (ne nieuwen $t v$, 'une nouvelle télévision', néerlandais standard : een nieuwe $t v)$, et laissent transparaître leur accent régional.

\section{En guise de conclusion (ironique) de l'histoire}

Ainsi, il est malheureusement classique de constater que l'histoire se répète : ce que les francophones disaient auparavant sur le néerlandais, les jugements en Flandre sur la tussentaal en particulier et sur l'allophonie en général (dont la francophonie) le redisent à leur tour. Toutefois,

33. Il est intéressant de savoir que le terme s'emploie aussi en français de Belgique, où il est fort productif : cokotter: 'partager un kot', cokotteur : 'cohabitant de kot', ... (cf. la Base de données lexicographiques panfrancophones : http://www.bdlp.org/). 
d'autres voix se font aussi entendre : la tussentaal serait «l'expression du statut dans la nouvelle riche Flandre ${ }^{34}$ ». L'auteur pose qu'elle est l'emblème de l'élite flamande qui a pleinement profité de l'essor économique de la région, qui a augmenté considérablement après la deuxième guerre mondiale. Arrivée en haut de la société flamande, cette élite peut maintenant se montrer indifférente à la norme prestigieuse et pratiquer l'hypocorrection ${ }^{35}$ en adoptant la tussentaal.

Comme l'indiquent Absillis, Jaspers \& Van Hoof (2012), la difficulté majeure reste toujours que la plupart des Flamands ne considèrent pas la tussentaal comme une variété à part, n'identifiant que la langue standard et le dialecte.

Il semble plausible que les descriptions linguistiques et sociolinguistiques qui se sont amplifiées à partir des années 2000, à la suite des travaux précurseurs de Kas Deprez dès les années 80, et les nouveaux usages pleinement assumés dans la société flamande puissent apporter la conscience de la tussentaal, norme endogène en Flandre, non pas uniquement pour l'oral colloquial, mais aussi plus largement pour le standard néerlandais belge à venir.

Je laisse enfin le mot de la fin à Kas Deprez :

J'oppose à la langue pannéerlandaise de Doornaert le flamandnéerlandais comme l'expression de mon identité belge. Mon héritage, c'est la Belgique, pas les Pays-Bas. Être belge, c'est vivre dans le même État que des locuteurs d'autres langues, un premier pas vers une citoyenneté européenne ${ }^{36}$.

34. Koen PleVoets, "Van hetzelfde laken een pak. Verkavelingsvlaams als uiting van status in het nieuwe rijke Vlaanderen", in Kevin Absillis, Jürgen Jaspers \& Sarah Van Hoof (dir.), De manke usurpator: over verkavelingsvlaams. Gent, Academia Press, 2012, p. 189-223.

35. Pierre Bourdieu, Ce que parler veut dire. L'économie des échanges linguistiques, Paris, Fayard, 1982, p. 54-55.

36. Kas DePrEZ, "Waarom ze hun Vlaams liever zien verdwijnen», Onze taal, 63, 11, 1994, p. 253. 


\section{Bibliographie}

ABSILLIS Kevin, JASPERS Jürgen, VAN HOOF Sarah (dir.), De manke usurpator. Over Verkavelingsvlaams, Gent, Academia Press, 2012, $430 \mathrm{p}$.

Bourdieu Pierre, Ce que parler veut dire. L'économie des échanges linguistiques, Paris, Fayard, 1982.

CAJOT José, «Waarom het Verkavelingsvlaams onvermijdelijk was. De ontwikkeling van een informele omgangstaal in Vlaanderen", in Kevin Absillis, Jürgen Jaspers \& Sarah Van Hoof (dir.), De manke usurpator. Over Verkavelingsvlaams, Gent, Academia Press, 2012, p. 39-66.

CLYNE Michael, "Pluricentricity: National Variety», in Ulrich Ammon (dir.), Status and Function of Languages and Language Varieties, Mouton de Gruyter, Berlin-New York, 1989, p. 357-371.

DE CALUWE Johan, «Tien stellingen over tussentaal in Vlaanderen", in Johan De Caluwe et al. (dir.), Taalvariatie en taalbeleid. AntwerpenApeldoorn, Garant, 2002, p. 57-67.

DE CALUWE Johan, "Tussentaal als natuurlijke omgangstaal in Vlaanderen", in Johan De Caluwe \& Magda Devos (dir.), Structuren in talige variatie in Vlaanderen, Gent, Academia Press, 2006, p. 19-34.

DE DeCKer Benny, VANDEKERCKHOVE Reinhild, "Stabilizing features in substandard Flemish: The chat language of Flemish teenagers as a test case ", Zeitschrift für Dialektologie und Linguistik, LXXIX, 79 (2), 2012, p. 129-148.

DE SCHRYVER Johan, «Het einde van de tussentaal en de Vlaamse standaardtaaldiscussie», in Kevin Absillis, Jürgen Jaspers \& Sarah Van Hoof (dir.), De manke usurpator: over verkavelingsvlaams. Gent, Academia Press, 2012, p. 141-165.

DEPREZ Kas, GEERTS Guido, "Lexical and pronominal standardization. The evolution of Standard Netherlandic in WestFlanders", in Zeitschrift für Dialektologie und Linguistik, Beihefte Neue Folge 22, 1977. 
DEPREZ Kas, Naar een eigen identiteit. Resultaten en evaluatie van tien jaar taalsociologisch en sociolinguistisch onderzoek betreffende de standaardtaal in Vlaanderen, Onuitgegeven proefschrift, Katholieke Universiteit Leuven, 1981.

DEPREZ Kas, Referentiekader voor het Vlaams Nederlands, De Standaard 9/10/1991.

DEPREZ Kas, «Waarom ze hun Vlaams liever zien verdwijnen», Onze taal, 63, 11, 1994, p. 251-253.

DEPREZ Kas, "Soldats du néerlandais", La Bretagne linguistique, vol. 10, actes du Colloque Badume-Standard-Norme : Le double jeu de la langue, Brest, UBO-CRBC, 1995, p. 189-215.

GEERAERTS Dirk, «Een zondagspak», Ons Erfdeel, 3 (44), 2001, p. 337-343.

GEERAERTS Rik, «Deprez-historie van het Vlaams», Yang, 37.4, 2001, p. 655-660.

HAESERYN Walter, "Grammaticale verschillen tussen het Nederlands in België en het Nederlands in Nederland: een poging tot inventarisatie», in Roeland Van Hout \& Joep Kruijsen (dir.), Taalvariaties. Toonzettingen en modulaties op een thema, Dordrecht, Foris, 1996, p. 109-126.

HINSKENS Frans, HEERINGA Wilbert, "Convergence between dialect varieties and dialect groups in the Dutch language area", in Benedikt Szmrecsanyi, Bernhard Wälchli (dir.), Aggregating dialectology, typology, and register analysis; linguistic variation in text and speech, Berlin-Boston, De Gruyter, 2014, p. 526-52.

HOPPENBROUWERS Cor, HOPPENBROUWERS Geer, «De featurefrequentiemethode en de classificatie van Nederlandse dialecten ", Tabu, 18, 1988, p. 51-92.

JASPAERT K., "Van oude normen, de dingen die voorbij gaan. Over de Nederlands-Vlaamse taalverhouding", Kultuurleven, 56.8, 1989, p. $16-23$

JASPERS Jeroen, BRISARD Frank, "Verklaringen van substandaardisering : tussentaal als gesitueerd taalgebruik", Leuvense Bijdrage, 2006, p. 35-70. 
JASPERS Jeroen, VAN HOOF Sarah, "Ceci n'est pas une tussentaal. Evoking standard and vernacular language through mixed Dutch in Flemish telecinematic discourse», Journal of Germanic Linguistics, 27.1, 2015.

JASPERS Jeroen, «Het Vlaamse stigma. Over tussentaal en normativiteit », Taal en Tongval, 53, 2001, p. 129-153.

LEDEGEN Gudrun, «Pour un néerlandais belge. Ecrits d'un précurseur. Introduction", in Jean Le Dû, Gudrun Ledegen, Pour un néerlandais de Belgique. Écrits d'un précurseur Hommage À Kas Deprez, Paris, L'Harmattan, Coll. "Espaces discursifs», 2019, p. 13-23.

LE DÛ Jean, LeDegen Gudrun, Pour un néerlandais de Belgique. Écrits d'un précurseur Hommage À Kas Deprez, Paris, L'Harmattan, Coll. «Espaces discursifs», 2019, 374 p.

PLEVOETS Koen, Tussen spreek-en standaardtaal. Een corpusgebaseerd onderzoek naar de situationele, regionale en sociale verspreiding van enkele morfosyntactische verschijnselen uit het gesproken BelgischNederlands, Leuven, Dissertation Doctorale, 2008.

PLEVOETS Koen, «Van hetzelfde laken een pak. Verkavelingsvlaams als uiting van status in het nieuwe rijke Vlaanderen ", in Kevin Absillis, Jürgen Jaspers \& Sarah Van Hoof (dir.), De manke usurpator: over verkavelingsvlaams, Gent, Academia Press, 2012, p. 189-223.

ROSIERS Kirsten, Tussen taal en tussentaal - De attitudes van leerlingen in het lager en middelbaar onderwijs tegenover talige variatie in Vlaanderen, Mémoire de Master, Universiteit Gent, 2009-2010.

SELINKER Larry, "Interlanguage», International Review of Applied Linguistics, 10, 1972, p. 219-231.

VANDEKERCKHOVE Reinhild, «Belgian Dutch versus Netherlandic Dutch : New patterns of divergence? On pronouns of address and diminutives ", Multilingua, 24, 2005, p. 379-397. 